\title{
Unmethylated Cytosine phosphate guanine DNA (CpG-DNA) exacerbates liver Kupffer cells (KCs) inflammation through Toll-like receptor 9 (TLR9) in diabetic rats
}

\section{Simin Cai}

Zhejiang University School of Medicine

Jing Li

Zhejiang University School of Medicine

Xiaoming Zhang ( $\boldsymbol{\nabla}$ zxm@zju.edu.cn )

Zhejiang University School of Medicine https://orcid.org/0000-0003-2292-4485

\section{Research Article}

Keywords: Diabetes mellitus, Gut microbiota, unmethylated CpG-DNA, KCs, Inflammation

Posted Date: March 12th, 2021

DOl: https://doi.org/10.21203/rs.3.rs-280189/v1

License: (c) (1) This work is licensed under a Creative Commons Attribution 4.0 International License.

Read Full License 


\section{Abstract}

Recently, gut microbiota for various pathogens has attracted attention. The present study investigated the role of gut microbiota unmethylated cytosine phosphate guanine DNA (CpG-DNA) on liver Kupffer cells (KCs) inflammatory cytokine interleukin-1 $\beta$ (IL-1 $\beta$ ) in diabetic rats. We induced diabetic rats models and sequenced the gut microbiota composition of fecal samples. We also applied CpG-DNA and TLR9 inhibitor on KCs to investigate the regulation of inflammatory cytokine IL-1 $\beta$ and Toll-like receptor 9 (TLR9) signaling pathway. We found a significant difference of gut microbiota between the control and the diabetic rats with increased Clostridium. Meanwhile, diabetes could upregulate TLR9 in KCs and increase IL-1 $\beta$ concentration. Furthermore, high concentration of unmethylated CpG-DNA could significantly increase IL-1 $\beta$ secretion while it was suppressed by TLR9 inhibitor in KCs cultured in high glucose medium. Our study suggests that unmethylated CpG-DNA, which was highly expressed in diabetic rats, activated KCs through TLR9, and induced IL-1 $\beta$ secretion in vitro and in vivo which plays an important role in diabetic liver inflammation. It may contribute to the progress of the diabetes.

\section{Introduction}

Diabetes mellitus (DM) is one of the most prevalent metabolic disorders which induced by both genetic and environment factors [1]. Accumulating evidences indicated that altered gut microbiota existed in patients with DM who had an increase of pathogens and a decrease of probiotics. It may contribute to impaired glucose tolerance and insulin signaling $[2,3]$. Pathogen and its metabolites such as lipopolysaccharide (LPS) and unmethylated cytosine phosphate guanine DNA (CpG-DNA) could reach the liver through the portal vein, arousing low-grade inflammation, while hyperglycemia drives intestinal barrier dysfunction which might contribute to the DM [4]. Unmethylated CpG-DNA is the specific component of bacterial DNA containing CpG motifs called pathogen associated molecular pattern (PAMP) [5]. The length, number and lateral sequence of unmethylated CpG-DNA that differ from different microbiota and determine their immunocompetence [6]. Pathogens such as Clostridia and Escherichia coli express higher unmethylated CpG-DNA that had stronger immunocompetence compared to the probiotics [7]. Recent study identified unmethylated CpG-DNA as a pathogen-dependent antigen that activated macrophage and induced inflammatory factors secretion[8]. PAMP can be specifically identified by pattern recognition receptor (PRR) including toll-like receptors (TLRs) in rats and human [5]. TLR9 is a major receptor of TLRs family that expresses in macrophage such as Kupffer cells (KCs) in liver and specifically recognizes unmethylated CpG-DNA $[9,10]$. Activated KCs secreted various proinflammatory cytokines including interleukin-1 $\beta$ (IL-1 $\beta$ ), a well-known inflammatory factor contributed to the low-grade inflammation and insulin resistance (IR) $[11,12]$. IL-1 $\beta$ over expression promoted phosphorylation and degradation of IRS, leading to the suppression of insulin signaling $[13,14]$ which promoted IR as well as the development of DM. However, the role of gut microbiota CpG-DNA on the development of DM is still elusive. The present study investigated the role of CpG-DNA on liver KCs to explore the possible mechanism of CpG-DNA activating KCs by TLR9 that induce liver inflammation in the progress of DM. 


\section{Materials And Methods}

\section{Experimental animal models}

Male SD rats (120-150g) (Shanghai laboratory animal center, Shanghai, China) were randomly divided into the control group (CT) and the diabetes mellitus group (DM). $24 \mathrm{~h}$ after fasting, the streptozotocin (65 mg/kg body weight, dissolved in $0.01 \mathrm{M}$ citrate buffer, $\mathrm{pH} 4.5$; Catalog: S8050, Solarbio, Beijing, China) were intraperitoneal injected to the rats of DM group while CT group were injected with equal volume of the vehicle. $72 \mathrm{~h}$ after diabetes conduction, the rats with blood glucose above $16.7 \mathrm{mmol} / \mathrm{L}$ were considered the successful diabetes models.

After six weeks, all rats were anesthetized by sodium pentobarbital ( $45 \mathrm{mg} / \mathrm{kg}$ body weight). Blood samples were obtained from hepatic portal vein to evaluate the level of IL-1 $\beta$. The liver and the body weight were also recorded. The liver of half rats in each group were removed and fixed in $4 \%$ paraformaldehyde solution overnight for hematoxylin-eosin (H\&E) and immunohistochemical staining, while another half liver in each group were preserved in liquid nitrogen for Western blot and ELISA assay.

\section{Gut microbiota analysis}

Fresh fecal samples were collected at the time of blood sampling, snap frozen in liquid nitrogen, and stored at $-80^{\circ} \mathrm{C}$ until analysis. Sequencing and data processing were conducted by lllumina MiSeq (Illumina, San Diego, USA). Microbial DNA was extracted from fecal samples using the E.Z.N.A.® soil DNA Kit (Omega Bio-tek, Norcross, GA, U.S.) according to manufacturer's protocols. Quantitative polymerase chain reaction was conducted using the following program: $3 \mathrm{~min}$ of denaturation at $95^{\circ} \mathrm{C}$, 27 cycles of $30 \mathrm{~s}$ at $95^{\circ} \mathrm{C}, 30 \mathrm{~s}$ for annealing at $55^{\circ} \mathrm{C}$, and $45 \mathrm{~s}$ for elongation at $72{ }^{\circ} \mathrm{C}$, and a final extension at $72{ }^{\circ} \mathrm{C}$ for 10 min by thermocycler PCR system (GeneAmp 9700, ABI, USA). PCR reactions were performed in triplicate $20 \mu \mathrm{L}$ mixture containing $4 \mu \mathrm{L}$ of $5 \times$ FastPfu Buffer, $2 \mu \mathrm{L}$ of $2.5 \mathrm{mM}$ dNTPs, $0.8 \mu \mathrm{L}$ of each primer $(5 \mu \mathrm{M}), 0.4 \mu \mathrm{L}$ of FastPfu Polymerase and $10 \mathrm{ng}$ of template DNA. The resulted PCR products were extracted from a $2 \%$ agarose gel and further purified using the AxyPrep DNA Gel Extraction Kit (Axygen Biosciences, Union City, CA, USA) and quantified using QuantiFluor ${ }^{\text {TM }}$-ST (Promega, USA) according to the manufacturer's protocol. Purified amplicons were pooled in equimolar and pairedend sequenced $(2 \times 300)$ on an Illumina MiSeq platform (Illumina, San Diego, USA) according to the standard protocols by Majorbio Bio-Pharm Technology Co. Ltd. (Shanghai, China). Raw fastq files were demultiplexed, quality-filtered by Trimmomatic and merged by FLASH with the following criteria: (i) The reads were truncated at any site receiving an average quality score $<20$ over a 50 bp sliding window. (ii) Primers were exactly matched allowing 2 nucleotide mismatching, and reads containing ambiguous bases were removed. (iii) Sequences whose overlap longer than $10 \mathrm{bp}$ were merged according to their overlap sequence. Operational taxonomic units (OTUs) were clustered with $97 \%$ similarity cutoff using UPARSE『version $7.1 \mathrm{http}: / /$ drive5.com/uparse/) and chimeric sequences were identified and removed using UCHIME. The taxonomy of each 16S rRNA gene sequence was analyzed by RDP Classifier 
algorithm (http://rdp.cme.msu.edu/) against the Silva (SSU123) 16S rRNA database using confidence threshold of $70 \%$.

\section{Hematoxylin-eosin staining (H\&E) and immunohistochemical staining}

The livers (in 4\% paraformaldehyde solution) were embedded in paraffin, then cut into $6-\mu \mathrm{m}$ section. The sections were respectively stained with H\&E to determine pathological changes. Images were observed by the microscope (BX-53, Olympus Corp, Tokyo, Japan).

The rest sections from each group were stained with immunohistochemical assay and incubated with the primary antibodies against TLR9 (TLR9, Catalog: ab134368, 1:200, Abcam, USA). After overnight incubation at $4{ }^{\circ} \mathrm{C}$, sections were washed with PBS $(3 \times 5 \mathrm{~min})$ and incubated with biotinylated and affinity-purified IgG secondary antibodies for $1 \mathrm{~h}$ at room temperature. Images were observed by the microscope (BX-53, Olympus Corp, Tokyo, Japan) and analyzed by Image-Pro Plus 6.0 (Media Cybernetics, USA).

\section{KCs were cultured with TLR9 agonist and inhibitor}

We used 1640 medium (Catalog: gnm31800, China) with 10\% (V/V) fetal bovine serum (Catalog: 10099141, Gibco, Invitrogen, USA) to culture KCs (Catalog: CP-R132, Procell Life Secience\&Technology, Wuhan, China) in the medium inside a tri-gas incubator (Thermo Fisher Scientific, Marietta, $\mathrm{OH}$, USA) composed of $5 \% \mathrm{CO} 2$ and $95 \%$ air at $37^{\circ} \mathrm{C}$.

$5 \times 10^{5} /$ well $\mathrm{KC}$ s were seeded in 6-well $(3 \mu \mathrm{M})$ system and divided into six groups: the control group (CT), the control group treated with unmethylated CpG-DNA (5.8 $\mu \mathrm{M}$, dissolved in $9.91 \mathrm{mM}$ DMSO; Catalog: HY101929, MedChemExpress, USA) (CT-OV), the control group treated with TLR9 inhibitor E6446 dihydrochloride (10 $\mu \mathrm{M}$, dissolved in $14.55 \mathrm{mM}$ DMSO; Catalog: HY-12756A, MedChemExpress, USA) (CT$\mathrm{SI}$ ), the diabetes group (DM), the diabetes group treated with unmethylated CpG-DNA (DM-OV) and the diabetes group treated with E6446 dihydrochloride (DM-SI). CT, CT-OV and CT-DM group were supplied with $5.5 \mathrm{mM}$ glucose, while DM, DM-OV and DM-SI group were supplied with $30 \mathrm{mM}$ high glucose to simulate the diabetic environment. After $24 \mathrm{~h}$ treatment, the cell culture supernatant from all groups were collected in the flow tube and centrifuged for $20 \mathrm{~min}$ at $1000 \times \mathrm{g}$ at $2 \sim 8{ }^{\circ} \mathrm{C}$ for ELISA assay. Cells were washed in PBS ( $3 \times 5 \mathrm{~min}$ ), lysed in Western \& IP buffer (Catalog: P0013, Beyotime Institute of Biotechnology, Shanghai, China) then used for Western blot assay.

\section{Western blot analysis}


The expression of proteins in liver and cultured cells were detected by Western blot assay. Equal amounts of protein were separated by $10 \%$ SDS-PAGE. It was transferred from the gel to nitrocellulose membrane, then blocked by $5 \%$ skimmed milk (Tris-buffered saline of $0.1 \%$ Tween 20 ) for $1 \mathrm{~h}$ at room temperature. The membranes were incubated with the primary antibodies (TLR9, Catalog: ab134368, 1:1000, Abcam, USA; IL-1 $\beta$, Catalog: ab9722, $0.8 \mu \mathrm{l} / \mathrm{ml}$, Abcam, USA) overnight at $4{ }^{\circ} \mathrm{C}$. The next day, the membranes were washed with TBST $(4 \times 5 \mathrm{~min})$ and incubated with infrared labeled secondary antibodies at room temperature for $1 \mathrm{~h}$. The immunoblotted bands were captured by a CLX Odyssey infrared imaging system (Li-COR biosciences, USA).

\section{ELISA analysis}

ELISA analysis of IL-1 $\beta$ levels in tissue homogenates, serum and cell culture supernatant of KCs were detected by Rat IL-1 $\beta$ ELISA Kit (Catalog: E-EL-R0012c, Elabscience, Wuhan, China). Sample collection, reagent preparation and assay procedure were strictly according to the kit instruction. The microplate reader (Thermo, USA) was used to assay the optical density (OD) values at $450 \mathrm{~nm}$.

\section{Statistical analysis}

The data are presented as mean \pm standard deviation (SD) and analyzed by SPSS 18.0 software (SPSS Inc., USA). The differences among both groups were performed with one-way analysis of variance (ANOVA). $P$ values $<0.05$ were considered statistically significant.

\section{Results}

\section{Gut microbiota changes in diabetic rats}

To validate the difference of gut microbiota communities, we investigated fetal samples from rats with or without DM. Results showed that bacteria diversity reduced in DM rats, compared to the normal rats (Fig. 1a). Abundance of different bacteria class (Fig. 1b), Sample distances heatmap (Fig. 1c) and hierarchical clustering tree (Fig. 1d) all presented significant difference between the control group and the diabetes group. The diabetic rats had more pathogens (such as Clostridiawere) and less probiotics (such as Bacteroidia and Gammaproteobacteria) than the normal rats $(\mathrm{P}<0.05$, vs control rats).

\section{Pathogen-dependent unmethylated CpG-DNA activates KCs through TLR9}

We adopted H\&E staining to confirm the inflammatory response in diabetic liver. We found inflammatory infiltration and cellular morphology changed in diabetic rats (Fig. 2a). Kupffer cells (KCs), the major macrophage in liver, playing an important role in inflammation. As a specific receptor of unmethylated 
CpG-DNA, immunofluorescent staining (Fig. 2b) and western blot assay (Fig. 2c-d) revealed that TLR9 was significantly increased by unmethylated CpG-DNA in DM rats ( $P<0.05, v s$ control rats). Thus, increasing TLR9 might present the activation of KCs in diabetic rats.

\section{TLR9 regulated KCs IL-1 $\beta$ secretion in virto and in vivo}

KCs activated initiate immune response for secreting a series of proinflammatory cytokines including IL$1 \beta$, a well-known proinflammatory cytokine. The western blot assay confirmed that an increased level of IL-1 $\beta$ in liver of DM rats, compared to the normal rats $(P<0.05$, Fig. $2 c, 3 a)$. ELISA analysis consistently showed the same results in both serum ( $\mathrm{P}<0.05$, Fig. $3 \mathrm{~b})$ and liver tissues (Fig.3c). In addition, ELISA assay showed that after 12 hours incubation with unmethylated CpG-DNA (CU-CPT17e), KCs significantly increased IL-1 $\beta$ expression. However, IL-1 $\beta$ expression was higher than the diabetic group without agonist treatment $(P<0.05)$. In contrast, E6446 dihydrochloride, an inhibitor of TLR9, suppressed IL-1 $\beta$ expression in the diabetic KCs significantly $(P<0.05$, Fig.3d). These results indicated that activated $K C$ s by unmethylated CpG-DNA increased IL-1 $\beta$ expression and inhibitor of TLR9 could reduce IL-1 $\beta$ secretion.

\section{Discussion}

Diabetes mellitus, characterized by glucose and insulin metabolism disturbances, is a rapidly increasing worldwide prevalence $[15,16]$. Recently, studies showed that gut microbiome was involved in various pathogens via generating bioactive metabolites[17]. Gut microbiota composition has a strong influence on weight gain and metabolic dysfunction [18]. Consistent with previous study [3]囚the present sequencing results revealed that the composition of gut microbiota in DM rats was different from the control rats, with significantly decreasing probiotics such as Bacteroidia and increasing pathogen such as Clostridia. Accordingly, several treatments based on supplement of probiotics also exhibited positive effects on DM patients $[19,20]$.

Pathogen and its metabolites might contribute to inflammation and diabetes development [4]. The studies have favored the roles of LPS in diabetes, and the results showed that LPS could initiate lowgrade inflammation and impair glucose tolerance [21,22]. However, as another major metabolite of pathogen, unmethylated CpG-DNA had the same capability as LPS [4]. Since unmethylated CpG-DNA is a specific component of bacteria DNA concentrating in pathogen, the concentration of unmethylated CpGDNA would increase with more pathogens and less probiotics in diabetic rats. $\mathrm{KCs}$, functioned as the dominant liver intravascular phagocytes, response to the pathogens and their metabolites[23]. In addition, as the specific receptor of unmethylated CpG-DNA, TLR9 was expressed in Kupffer cells囚providing a solid foundation for activation of KCs by unmethylated CpG-DNA, and induction of interleukin-1 $\beta$ in mice [11].

The present study confirmed that liver inflammation occurred and altered gut microbiota composition in diabetic rats. Interestingly, we found that diabetes promoted expression of TLR9 in KCs. As we know, KCs were the major source of IL-1 $\beta$ in liver [12] and played an important role in inflammation and DM 
development $[13,14]$. Increasing IL-1 $\beta$ could down regulate PI3K/Akt insulin signal pathway and lead to the progress of DM. We found that IL-1 $\beta$ was not only present at higher concentration in portal blood and liver tissue in DM rats, but in the cultured KCs treated by high concentration unmethylated CpG-DNA. Furthermore, TLR9 inhibitor treatment could suppress the CpG-DNA induced IL-1 $\beta$ secretion. The present data suggested that upregulation of TLR9 could activate KCs to promote inflammatory response and $1 \beta$ secretion which may play a role in DM development.

In conclusion, the present results demonstrated that gut microbiota unmethylated CpG-DNA could activate TLR9 of KCs and increase IL-1 $\beta$ secretion in the diabetic liver and cultured $\mathrm{KCs}$, leading to liver inflammation. It may contribute to the DM development. The current study for the first time provided a potential target for the therapy of DM with improving gut microbiota.

\section{Declarations}

\section{Funding:}

The study is supported by the Natural Science Foundation of Zhejiang Province China (LY18H170002) and the National Natural Science Foundation of China (81472149).

\section{Conflict of Interest:}

The authors declare that there are no conflicts of interest.

\section{Availability of data and material:}

The datasets used or analyzed during the current study are available from the corresponding author on reasonable request.

\section{Code availability:}

Not applicable.

\section{Authors contributions:}

All the authors contributed to conception and study design. Cai contributed to acquisition of the data, Li and Cai contributed to analysis of the data. All the authors contributed to drafting and critical revision of the manuscript of important intellectual content. All the authors have given final approval of the version to be published and agreed to be accountable for all aspects of the work.

\section{Ethics approval[}

The animal experiments were approved by the Committee of Animal Experiment Center of Zhejiang University (Hangzhou, China) which complies with the guidelines of the National Institutes of Health guide for the care and use of Laboratory animals (NIH Publications No. 8023, revised 1978). 


\section{Consent to participate:}

Not applicable.

\section{Consent for publication:}

Written informed consent for publication was obtained from all participants.

\section{Acknowledgements}

The study is supported by the Natural Science Foundation of Zhejiang Province China (LY18H170002) and the National Natural Science Foundation of China (81472149).

\section{References}

1. Peres BU, Hirsch Allen AJ, Fox N, Laher I, Hanly P, Skomro R, Almeida F, Ayas NT, Canadian S and Circadian N (2019) Circulating biomarkers to identify cardiometabolic complications in patients with Obstructive Sleep Apnea: A systematic review. Sleep Med Rev 44:48-57. doi: 10.1016/j.smrv.2018.12.004

2. Just S, Mondot S, Ecker J, Wegner K, Rath E, Gau L, Streidl T, Hery-Arnaud G, Schmidt S, Lesker TR, Bieth V, Dunkel A, Strowig T, Hofmann T, Haller D, Liebisch G, Gerard P, Rohn S, Lepage P and Clavel T (2018) The gut microbiota drives the impact of bile acids and fat source in diet on mouse metabolism. Microbiome 6:134. doi: 10.1186/s40168-018-0510-8

3. Zhao L, Zhang F, Ding X, Wu G, Lam YY, Wang X, Fu H, Xue X, Lu C, Ma J, Yu L, Xu C, Ren Z, Xu Y, Xu S, Shen H, Zhu X, Shi Y, Shen Q, Dong W, Liu R, Ling Y, Zeng Y, Wang X, Zhang Q, Wang J, Wang L, Wu $Y$, Zeng B, Wei H, Zhang M, Peng $Y$ and Zhang C (2018) Gut bacteria selectively promoted by dietary fibers alleviate type 2 diabetes. Science 359:1151-1156. doi: 10.1126/science.aao5774

4. Thaiss CA, Levy M, Grosheva I, Zheng D, Soffer E, Blacher E, Braverman S, Tengeler AC, Barak O, Elazar M, Ben-Zeev R, Lehavi-Regev D, Katz MN, Pevsner-Fischer M, Gertler A, Halpern Z, Harmelin A, Aamar S, Serradas P, Grosfeld A, Shapiro H, Geiger B and Elinav E (2018) Hyperglycemia drives intestinal barrier dysfunction and risk for enteric infection. Science 359:1376-1383. doi: 10.1126/science.aar3318

5. Patra MC, Shah M and Choi S (2020) Toll-like receptor-induced cytokines as immunotherapeutic targets in cancers and autoimmune diseases. Semin Cancer Biol 64:61-82. doi: 10.1016/j.semcancer.2019.05.002

6. Clauson RM, Berg B and Chertok B (2019) The Content of CpG-DNA in Antigen-CpG Conjugate Vaccines Determines Their Cross-Presentation Activity. Bioconjug Chem 30:561-567. doi: 10.1021/acs.bioconjchem.9b00091

7. Ohto U, Shibata T, Tanji H, Ishida H, Krayukhina E, Uchiyama S, Miyake K and Shimizu T (2015) Structural basis of CpG and inhibitory DNA recognition by Toll-like receptor 9. Nature 520:702-5. doi: $10.1038 /$ nature 14138 
8. Abdul-Cader MS, Amarasinghe A, Palomino-Tapia V, Ahmed-Hassan H, Bakhtawar K, Nagy E, Sharif S, Gomis S and Abdul-Careem MF (2018) In ovo CpG DNA delivery increases innate and adaptive immune cells in respiratory, gastrointestinal and immune systems post-hatch correlating with lower infectious laryngotracheitis virus infection. PLoS One 13:e0193964. doi:

10.1371/journal.pone.0193964

9. Teratani T, Tomita K, Suzuki T, Furuhashi H, Irie R, Hida S, Okada Y, Kurihara C, Ebinuma H, Nakamoto N, Saito H, Hibi T, Miura S, Hokari R and Kanai T (2017) Free cholesterol accumulation in liver sinusoidal endothelial cells exacerbates acetaminophen hepatotoxicity via TLR9 signaling. J Hepatol 67:780-790. doi: 10.1016/j.jhep.2017.05.020

10. Maatouk L, Compagnion AC, Sauvage MC, Bemelmans AP, Leclere-Turbant S, Cirotteau V, Tohme M, Beke A, Trichet M, Bazin V, Trawick BN, Ransohoff RM, Tronche F, Manoury B and Vyas S (2018) TLR9 activation via microglial glucocorticoid receptors contributes to degeneration of midbrain dopamine neurons. Nat Commun 9:2450. doi: 10.1038/s41467-018-04569-y

11. Miura K, Kodama Y, Inokuchi S, Schnabl B, Aoyama T, Ohnishi H, Olefsky JM, Brenner DA and Seki E (2010) Toll-like receptor 9 promotes steatohepatitis by induction of interleukin-1beta in mice. Gastroenterology 139:323-34 e7. doi: 10.1053/j.gastro.2010.03.052

12. Marra F and Tacke F (2014) Roles for chemokines in liver disease. Gastroenterology 147:577-594 e1. doi: $10.1053 /$ j.gastro.2014.06.043

13. Khodabandehloo H, Gorgani-Firuzjaee S, Panahi G and Meshkani R (2016) Molecular and cellular mechanisms linking inflammation to insulin resistance and beta-cell dysfunction. Transl Res 167:228-56. doi: 10.1016/j.trsl.2015.08.011

14. Wada $\mathrm{J}$ and Makino $\mathrm{H}$ (2016) Innate immunity in diabetes and diabetic nephropathy. Nat Rev Nephrol 12:13-26. doi: 10.1038/nrneph.2015.175

15. Multhaup ML, Seldin MM, Jaffe AE, Lei X, Kirchner H, Mondal P, Li Y, Rodriguez V, Drong A, Hussain M, Lindgren C, McCarthy M, Naslund E, Zierath JR, Wong GW and Feinberg AP (2015) Mouse-human experimental epigenetic analysis unmasks dietary targets and genetic liability for diabetic phenotypes. Cell Metab 21:138-49. doi: 10.1016/j.cmet.2014.12.014

16. Moelands SV, Lucassen PL, Akkermans RP, De Grauw WJ and Van de Laar FA (2018) Alphaglucosidase inhibitors for prevention or delay of type 2 diabetes mellitus and its associated complications in people at increased risk of developing type 2 diabetes mellitus. Cochrane Database Syst Rev 12:CD005061. doi: 10.1002/14651858.CD005061.pub3

17. Wu K, Yuan Y, Yu H, Dai X, Wang S, Sun Z, Wang F, Fei H, Lin Q, Jiang H and Chen T (2020) The gut microbial metabolite trimethylamine $\mathrm{N}$-oxide aggravates GVHD by inducing M1 macrophage polarization in mice. Blood 136:501-515. doi: 10.1182/blood.2019003990

18. Robertson RC, Kaliannan K, Strain CR, Ross RP, Stanton C and Kang JX (2018) Maternal omega-3 fatty acids regulate offspring obesity through persistent modulation of gut microbiota. Microbiome 6:95. doi: 10.1186/s40168-018-0476-6 
19. Mobini R, Tremaroli V, Stahlman M, Karlsson F, Levin M, Ljungberg M, Sohlin M, Berteus Forslund H, Perkins R, Backhed F and Jansson PA (2017) Metabolic effects of Lactobacillus reuteri DSM 17938 in people with type 2 diabetes: A randomized controlled trial. Diabetes Obes Metab 19:579-589. doi: 10.1111/dom. 12861

20. Raygan F, Ostadmohammadi V, Bahmani F and Asemi Z (2018) The effects of vitamin D and probiotic co-supplementation on mental health parameters and metabolic status in type 2 diabetic patients with coronary heart disease: A randomized, double-blind, placebo-controlled trial. Prog Neuropsychopharmacol Biol Psychiatry 84:50-55. doi: 10.1016/j.pnpbp.2018.02.007

21. Langston PK, Nambu A, Jung J, Shibata M, Aksoylar HI, Lei J, Xu P, Doan MT, Jiang H, MacArthur MR, Gao X, Kong Y, Chouchani ET, Locasale JW, Snyder NW and Horng T (2019) Glycerol phosphate shuttle enzyme GPD2 regulates macrophage inflammatory responses. Nat Immunol 20:1186-1195. doi: 10.1038/s41590-019-0453-7

22. Tanaka H, Nishikawa Y, Fukushima T, Taniguchi A, Fujita Y, Tsuda K, Inagaki N and Hosokawa M (2017) Lipopolysaccharide inhibits hepatic gluconeogenesis in rats: The role of immune cells. J Diabetes Investig. doi: 10.1111/jdi.12729

23. Triantafyllou E, Gudd CLC, Mawhin MA, Husbyn HC, Trovato FM, Siggins MK, O'Connor T, Kudo H, Mukherjee SK, Wendon JA, Bernsmeier C, Goldin RD, Botto M, Khamri W, McPhail MJ, Possamai LA, Woollard KJ, Antoniades CG and Thursz MR (2020) PD-1 blockade improves Kupffer cell bacterial clearance in acute liver injury. J Clin Invest. doi: 10.1172/JCI140196

\section{Figures}


A

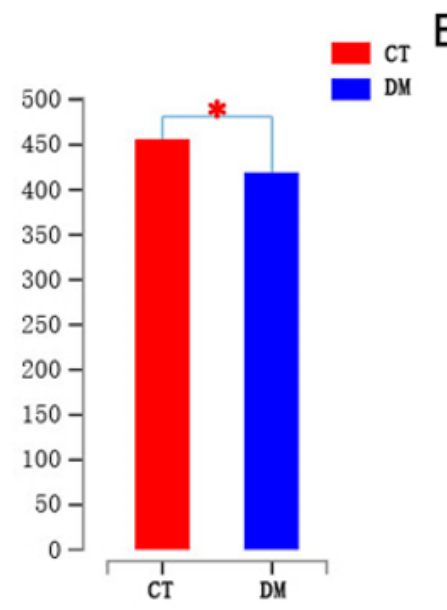

B

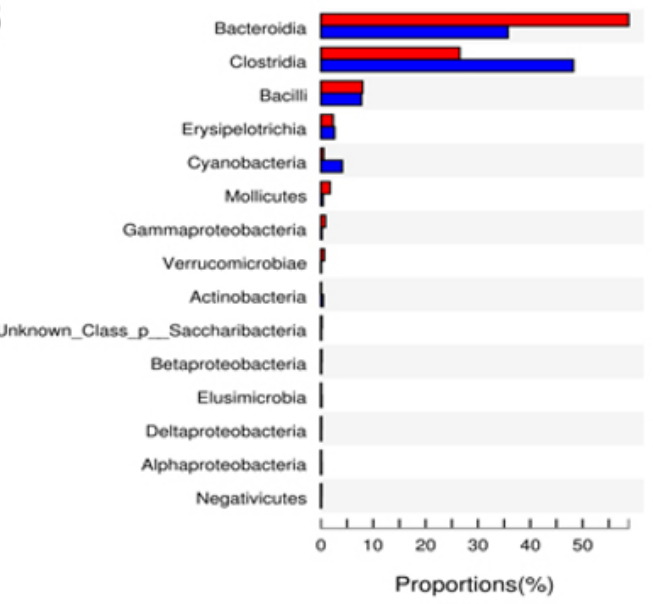

$95 \%$ confidence intervals

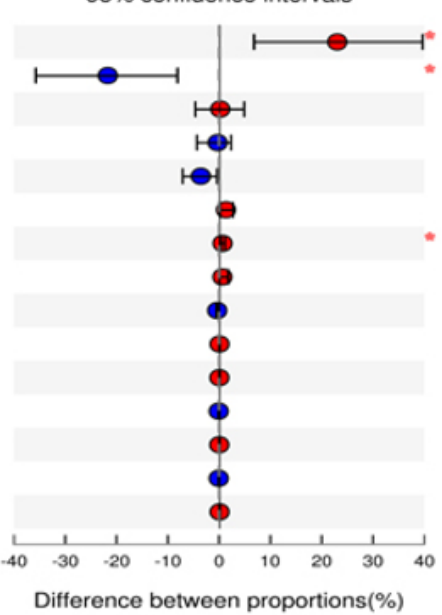

- CT

0.03576 0.02248 0.2353

0.7842 0.08284

0.02248 0.06166 0.08284 0.1709 0.1207

0.1709

0.05523

0.4113

0.3153
C

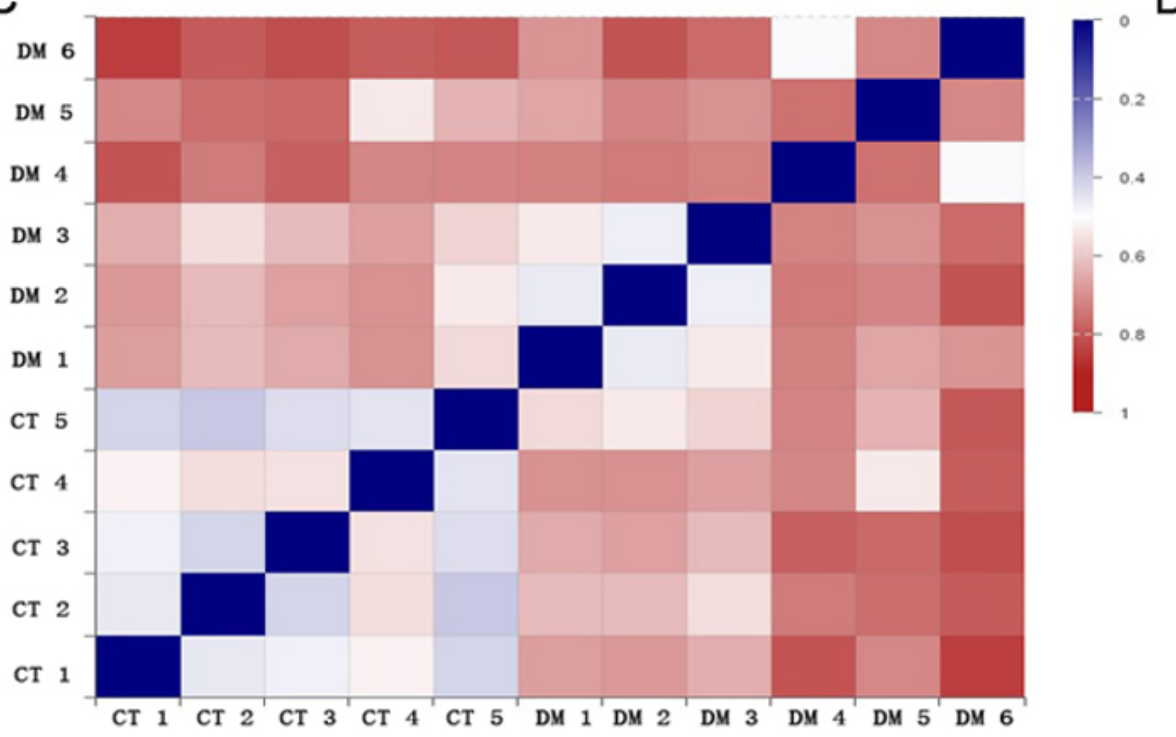

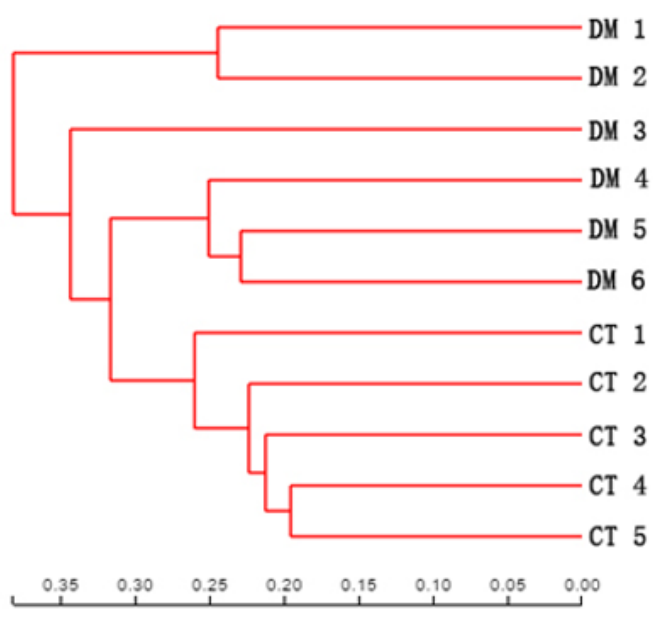

Figure 1

Difference of gut microbiota composition in two groups. (a) The diversity of total gut microbiota. (b) Abundance of bacteria in both groups at the class level. Bacterial strains that were significantly higher or lower are shown as red or blue bars, respectively. (c) Sample distances heatmap. (d) Hierarchical clustering tree of gut microbiota. All data are presented as mean $\pm S D ;{ }^{*}<<0.05$, vs CT group $(n=5)$. 
A
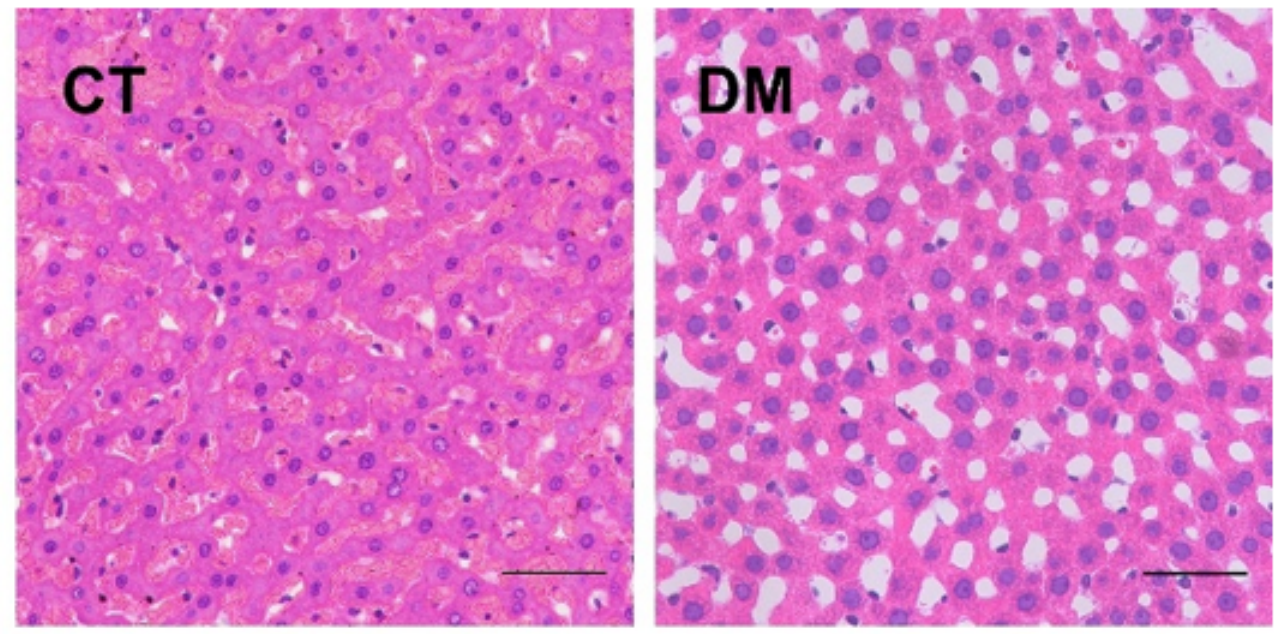

B
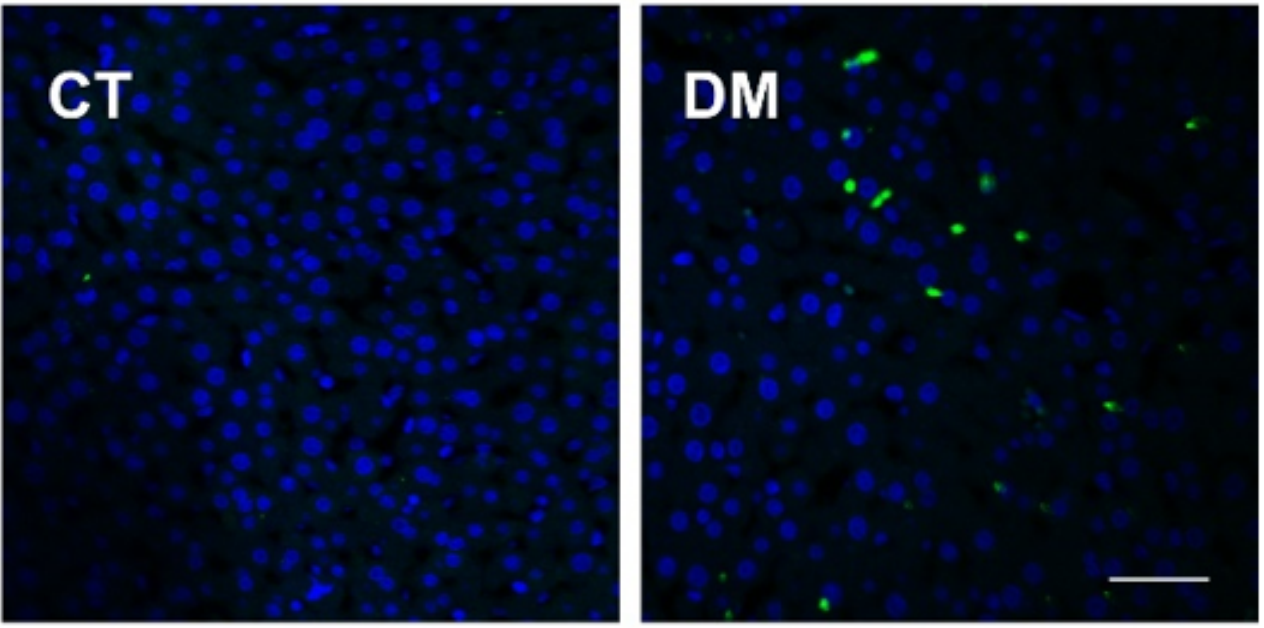

C
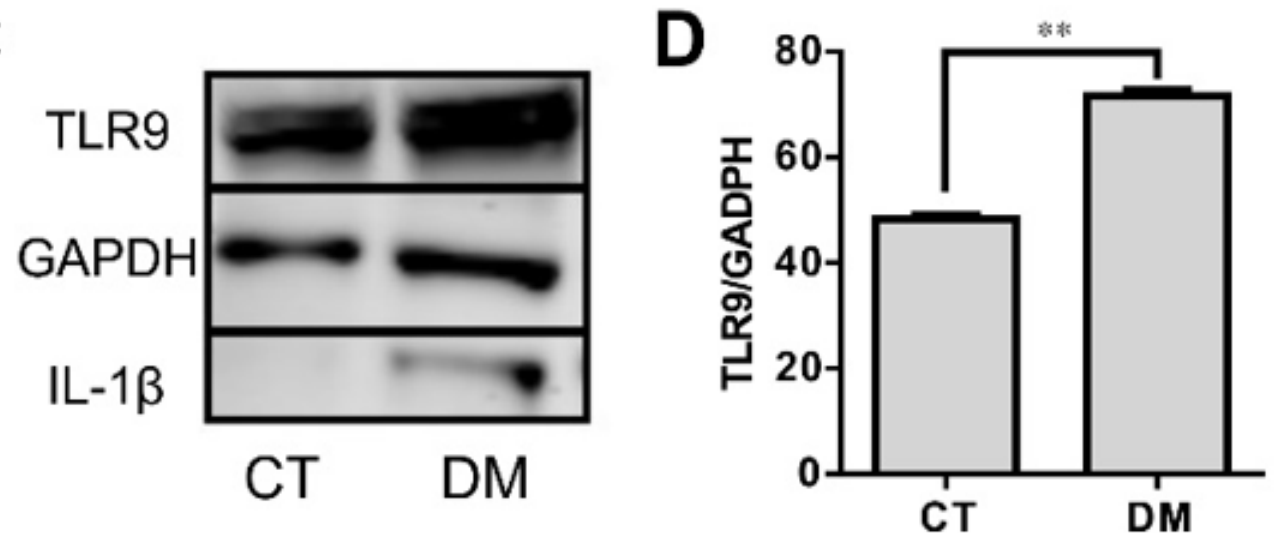

Figure 2

TLR9 increased in liver of two groups. (a) HE staining images of liver for inflammatory response. Scale bar: $50 \mu \mathrm{m}$. (b) Representative images of immunofluorescence staining for TLR9. Scale bar: $50 \mu \mathrm{m}$. (c) Western blotting result of liver tissue. (d) Quantitation of TLR9 by western blotting. Data are presented as mean \pm SD. ${ }^{* *} p<0.01$. $(n=3)$. 

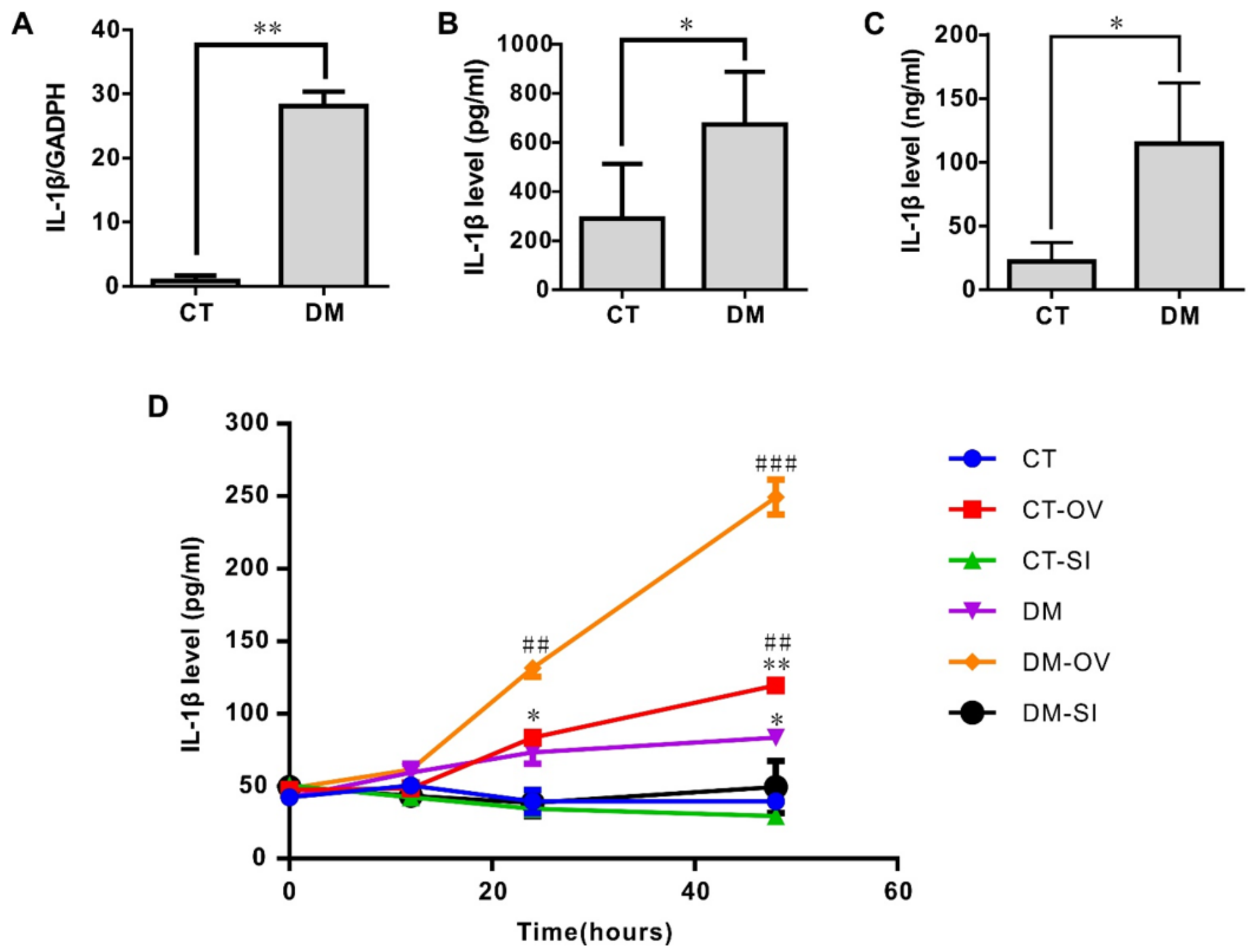

Figure 3

Expression of IL-1 $\beta$ in serum and liver of T2DM rats. (a) Quantitation of IL-1 $\beta$ in liver by western blotting. ${ }^{* \star} p<0.01$. (b) The ELISA assay of IL-1 $\beta$ level in liver tissue. (c) The ELISA assay of IL-1 $\beta$ level in serum. (d) The ELISE assay of IL-1 $\beta$ secreted by KCs treated with TLR9 agonist or inhibitor. CT: the control group; CTOV: the control group treated with TLR9 agonist CU-CPT17e; CT-SI: the control group treated with TLR9 inhibitor E6446 dihydrochloride; DM: the diabetes group; DM-OV: the diabetes group treated with CUCPT17e; DM-SI: the diabetes group treated with E6446 dihydrochloride. ${ }^{*} p<0.05$, ${ }^{*} p<0.01$, vs CT group; $\# p<0.05, \# \# p<0.01, \# \# \# p<0.001$, vs $D M$ group. All data are presented as mean $\pm S D,(n=3)$. 


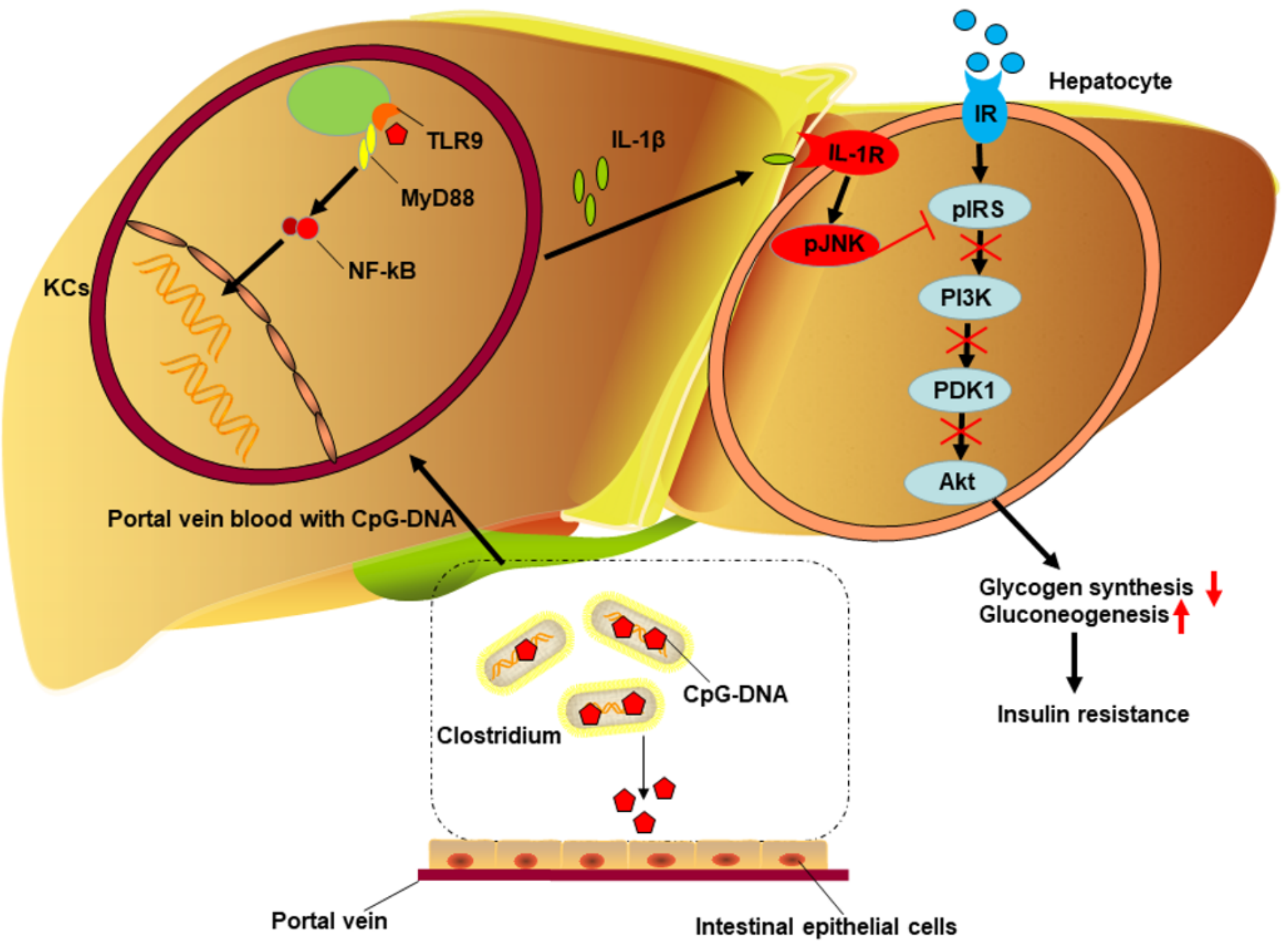

Figure 4

Graphical abstract of this study. 